\title{
On Galerkin Difference Methods
}

\author{
J. W. Banks ${ }^{\mathrm{a}, 1, *}$, T. Hagstrom ${ }^{\mathrm{b}, 1}$ \\ ${ }^{a}$ Department of Mathematical Sciences, Rensselaer Polytechnic Institute, Troy, NY USA \\ ${ }^{b}$ Department of Mathematics, Southern Methodist University, PO Box 750156, Dallas, TX 75275-0156, USA
}

\begin{abstract}
Energy-stable difference methods for hyperbolic initial-boundary value problems are constructed using a Galerkin framework. The underlying basis functions are Lagrange functions associated with continuous piecewise polynomial approximation on a computational grid. Both theoretical and computational evidence shows that the resulting methods possess excellent dispersion properties. In the absence of boundaries the spectral radius of the operators for the first and second derivative matrices are bounded independent of discretization order. With boundaries the spectral radius of the first order derivative matrix appears to be bounded independent of discretization order, and grows only slowly with discretization order for problems in second-order form.
\end{abstract}

Keywords: difference methods, Galerkin methods, initial-boundary value problems

\section{Introduction}

It is well established that high-order accurate schemes (e.g., fourth-order, sixth-order or higher) can be orders of magnitude faster and more efficient than their low-order counterparts, although the development of robust and efficient high-order schemes for complex geometry can be difficult. In addition, schemes for purely hyperbolic equations such as those found in acoustics, elasticity, or electrodynamics must address the fact that the partial differential equations (PDEs) in question possess no natural dissipation. Methods based on summation-by-parts operators or variational methods are attractive since energy-conserving and non-dissipative discrete approximations can be developed, as well as upwind schemes with parameter-free dissipation mechanisms. Summation-by-parts (SBP) methods [1, 2, 3] have been well studied, and, while having many attractive features, they have limitations such as reduced accuracy near boundaries and restricted applicability for general grids and geometries. Variational-methods have a very significant history with the vast majority of work being on finite element $(\mathrm{FE})$ methods $[4,5]$. At one end of the variational spectrum are high-order p-refinement $[6,7]$ and spectral-element [8] methods that achieve high orders of accuracy by adding interior degrees of freedom (DOF) to each element. At the other end of the spectrum are spline-based FE methods [9] that qbtin more accurate approximations by imposing higher degrees of continuity at element boundaries, but do not introduce additional interior degrees of freedom.

In this paper we study a new class of discretization schemes which we call Galerkin Difference Methods (GD). These methods do not introduce interior degrees of freedom and obtain high order accuracy by including neighboring DOFs while imposing only low-order continuity at element boundaries [10]. In this sense GD bears a resemblance to high-order finite-difference methods, and indeed away from boundaries the GD scheme resembles a compact finite difference scheme [11]. The fundamental idea of the GD method is based on the observation that in defining a continuous representation of a given discrete dataset over a

${ }^{*}$ Corresponding author.

Email addresses: banksj3@rpi.edu (J. W. Banks), thagstrom@smu.edu (T. Hagstrom)

${ }^{1}$ This work was supported by contracts from the U.S. Department of Energy ASCR Applied Math Program

${ }^{2}$ This work was supported by contracts from the U.S. Department of Energy ASCR Applied Math Program, ARO Grant W911NF-09-1-0344 and NSF Grant DMS-1418871. Any opinions, findings, and conclusions or recommendations expressed in this material are those of the authors and do not necessarily reflect the views of the Department of Energy, the Army Research Office or the National Science Foundation. 
domain, one has implicitly employed a set of basis functions. This set of basis functions can be used in a Galerkin projection technique to define energy stable PDE discretizations. Key features of GD discretizations include:

1. Away from domain boundaries, the discrete approximations are uniform from grid-point to grid-point. By contrast in p-refinement methods there are generally different equations, and therefore different local truncation errors, associated with each DOF.

2. No new DOFs are added as the approximation order increases. Therefore, memory requirements do not increase with order.

3. Explicitly integrated GD approximations do not require significantly smaller time-steps (i.e., smaller Courant-Friedrichs-Lewy (CFL) number) with increasing order; indeed for first-order systems no significant CFL penalty is observed. This is in contrast to typical p-refinement FE approximations where the maximal stable time step scales inversely with approximation order so that smaller CFL numbers are needed as the order of accuracy increases 12 .

The focus of the current manuscript is the basic construction and properties of the schemes in one space dimension. Away from boundaries GD discretizations will be shown to have the structure of a form of compact finite difference scheme. However, as noted in 13 it is unclear if compact finite differences can be directly formulated within an SBP framework, and in the current work provably energy stable boundary closures are obtained by employing a Galerkin projection. Here we choose a standard continuous Galerkin discretization of the second order scalar wave equation, and a Galerkin discretization of first order systems with an upwinded implementation of the boundary conditions. The latter can be viewed as a standard discontinuous Galerkin discretization on a domain with a single element, or as an SBP scheme with a penalty implementation of the boundary conditions. However we emphasize that the bases we construct can be used in any Galerkin framework.

Note that in the seventies Wendroff and coworkers studied, in a finite difference context, Galerkin methods based on B-spline basis functions 14, 15. These bear many similarities to the methods we propose here. In particular the B-spline methods produce energy stable compact difference approximations with mass matrices of the same bandwidth, and their methods also exhibit superconvergence away from boundaries at the same order as ours. Interestingly, they must reinterpret the B-spline expansion coefficients as nodal values to obtain these results, whereas the methods proposed here require no such reinterpretation. As indicated in this manuscript, there are numerous other possibilities for defining the piecewise polynomial basis functions, and we plan to make a serious comparison of some of these choices in subsequent work.

The paper is organized as follows. In Section 2 we introduce the basis functions we propose to use. They are simply Lagrange functions associated with piecewise degree $p$ polynomial interpolants constructed from data on a uniform grid. They differ from a standard finite element basis as the interpolant on a given cell involves data from outside the cell. In Section 3 we discuss the behavior and possible modification of the basis functions near domain boundaries. Our methods are all constructed using ghost nodes as a device, with different constructions following from leaving the associated ghost data free or relating it to internal data by extrapolation or higher order compatibility with the governing equation and boundary conditions. In Section 4 we apply the method to the wave equation in second order form. We show that the dispersion error of the scheme away from boundaries converges at almost twice the design order, quantify the mild growth with order of the spectral radius of the discrete wave operator with increasing order, and carry out a simple numerical experiment to study convergence. In Section 5 we do the same for approximations to symmetric hyperbolic systems in first-order form. Here the dispersion error away from boundaries is even more rapidly convergent, and the growth with increasing order of the spectral radius of the discrete derivative operator is found to be negligible. Finally in Sections 6 and 7 we discuss the extension of the method to higher space dimension and to more complex settings. Experiments with these extensions will appear elsewhere.

\section{Basis Functions}

In order to introduce our new approach, begin by considering a free space problem so that no boundary modifications are present. Boundary closures will be treated later in Section 3. Assume $x_{j}=j h$ where $j=\ldots,-1,0,1, \ldots$, and $h$ is a uniform grid spacing. Furthermore, assume that $u_{j} \approx u\left(x_{j}\right)$ is a discrete 
approximation of the continuous function at the grid points. Our approximation of $u(x)$, denoted $\tilde{u}(x)$, over each interval $\left(x_{k}, x_{k+1}\right)$ will simply be the degree- $p$ polynomial interpolants of the nodal data at $p+1$ consecutive points including $x_{k}$ and $x_{k+1}$. Although the construction and analysis of the methods applies to general interpolation schemes, we restrict our attention here to the most obvious choice: $p=2 q-1$ odd and the symmetric $2 q$-point stencil $x_{k-q+1}, \ldots, x_{k+q}$. Then

$$
\tilde{u}(x)=\sum_{\alpha=k-q+1}^{k+q} u_{\alpha} l_{\alpha}^{(p)}(x), \quad \text { for } x_{k}<x \leq x_{k+1}
$$

where $l_{\alpha}^{(p)}$ are the Lagrange basis polynomials of degree $p$. Considering a collection of nearby intervals, each $u_{j}$ will appear as a coefficient in the interpolating polynomial for $p+1=2 q$ intervals. Therefore, basis functions, $\phi_{j}^{(p)}(x)$ can be associated with each $x_{j}$ with the property that each basis function is nonzero only over each of the $p+1$ neighboring intervals. Piecewise linear reconstruction $(p=1)$ will yield the well known-piecewise linear basis functions which are a standard choice for finite element approximations. However, unlike the usual construction of higher degree finite element bases, our higher degree basis functions span a number of intervals.

As an example, for $p=3$ the support of a basis function will span four cells. An explicit formula is

$$
\phi_{j}^{(3)}\left(\xi_{j}\right)=\left\{\begin{array}{cc}
\frac{\left(\xi_{j}+3 h\right)\left(\xi_{j}+2 h\right)\left(\xi_{j}+h\right)}{6 h^{3}} & -2 h<\xi_{j} \leq-h \\
-\frac{\left(\xi_{j}+2 h\right)\left(\xi_{j}+h\right)\left(\xi_{j}-h\right)}{2 h^{3}} & -h<\xi_{j} \leq 0 \\
\frac{\left(\xi_{j}+h\right)\left(\xi_{j}-h\right)\left(\xi_{j}-2 h\right)}{2 h^{3}} & 0<\xi_{j} \leq h \\
-\frac{\left(\xi_{j}-h\right)\left(\xi_{j}-2 h\right)\left(\xi_{j}-3 h\right)}{6 h^{3}} & h<\xi_{j} \leq 2 h \\
0 & \text { else }
\end{array}\right.
$$

where $\xi_{j}=x-x_{j}$.

Similarly for $p=5$

$$
\phi_{j}^{(5)}\left(\xi_{j}\right)=\left\{\begin{array}{cc}
\frac{\left(\xi_{j}+5 h\right)\left(\xi_{j}+4 h\right)\left(\xi_{j}+3 h\right)\left(\xi_{j}+2 h\right)\left(\xi_{j}+h\right)}{120 h^{5}} & -3 h<\xi_{j} \leq-2 h \\
-\frac{\left(\xi_{j}+4 h\right)\left(\xi_{j}+3 h\right)\left(\xi_{j}+2 h\right)\left(\xi_{j}+h\right)\left(\xi_{j}-h\right)}{24 h^{5}} & -2 h<\xi_{j} \leq-h \\
\frac{\left(\xi_{j}+3 h\right)\left(\xi_{j}+2 h\right)\left(\xi_{j}+h\right)\left(\xi_{j}-h\right)\left(\xi_{j}-2 h\right)}{12 h^{5}} & h<\xi_{j} \leq 0 \\
-\frac{\left(\xi_{j}+2 h\right)\left(\xi_{j}+h\right)\left(\xi_{j}-h\right)\left(\xi_{j}-2 h\right)\left(\xi_{j}-3 h\right)}{12 h^{5}} & 0<\xi_{j} \leq h \\
\frac{\left(\xi_{j}+h\right)\left(\xi_{j}-h\right)\left(\xi_{j}-2 h\right)\left(\xi_{j}-3 h\right)\left(\xi_{j}-4 h\right)}{2 h^{5}} & h<\xi_{j} \leq 2 h \\
-\frac{\left(\xi_{j}-h\right)\left(\xi_{j}-2 h\right)\left(\xi_{j}-3 h\right)\left(\xi_{j}-4 h\right)\left(\xi_{j}-5 h\right)}{120 h^{5}} & 2 h<\xi_{j} \leq 3 h \\
0 & \text { else. }
\end{array}\right.
$$

These basis functions, as well as those obtained with $p=7$ and $p=9$, are plotted in Figure 1. Of note is the rapid decay of the functions away from $\xi_{j}$ which will imply the rapid decay of the elements of the mass, stiffness, and derivative matrices off the diagonal. Also we observe that the basis functions approach the spectral basis $\operatorname{sinc}(x)$ as the polynomial order $p$ approaches infinity.

\section{Boundary closures}

The construction of the basis elements discussed above must clearly be modified near boundaries. A number of approaches are possible and a distinct advantage of the Galerkin approach is that any method we choose will lead to energy stable discretizations. However, the conditioning and accuracy of the mass, stiffness, and derivative matrices will be strongly dependent on the modifications chosen. Here we will pursue a method inspired by standard practice in the finite difference community, namely the introduction of so-called ghost nodes outside of the physical domain. For clarity, consider the interval $[a, b]$ with $x_{0}=a$, $x_{N}=b$ located on the boundary. Then $x_{j}=j h, j=-1, \ldots, 1-q$ and $j=N+1, \ldots, N+q-1$ are ghost nodes located outside $[a, b]$. The basis functions $\phi_{j}^{(p)}(x)$ with $j<0$ will be used in the approximation to $u$ 

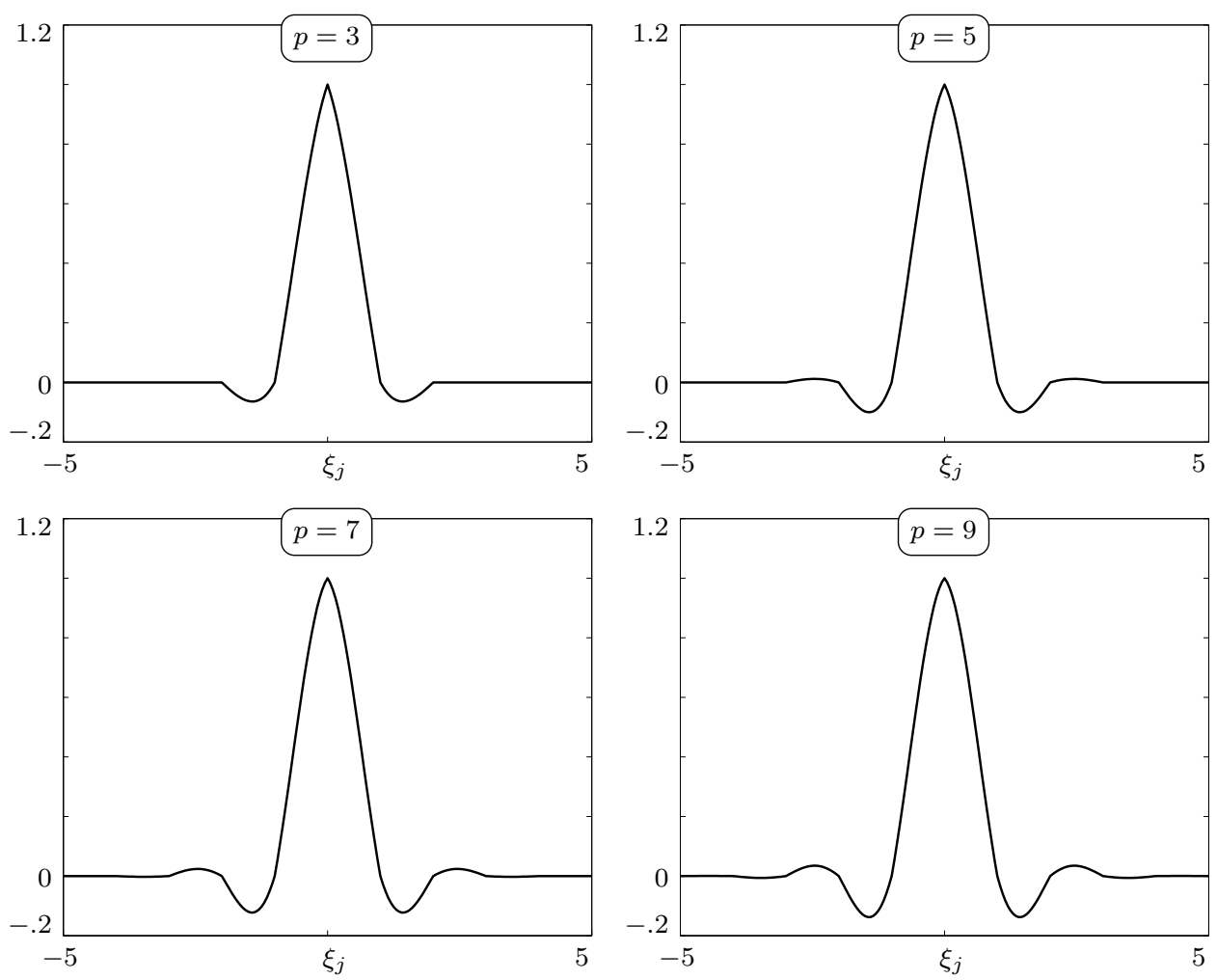

Figure 1: Basis functions.

in the cells near $x=a$ and those with $j>N$ will contribute near $x=b$. The issue is how to incorporate the degrees-of-freedom associated with these basis functions (i.e. the "values" of $u$ outside the domain) into the discrete equations. We will examine three distinct methods for accomplishing this - two generic approaches, denoted ghost basis and extrapolation, and one which uses the governing equation and boundary condition to derive extra compatibility conditions, denoted compatibility. When feasible we find that the latter approach can have significant advantages in terms of the maximum eigenvalue of the operators as well as the accuracy of the resulting schemes, but its application in general multidimensional settings is challenging.

\subsection{Generic Boundary Closures}

Ghost Basis: Perhaps the most straightforward approach is to simply include the basis functions, $\phi_{j}^{(p)}$, $j<0, j>N$, in the definition of the trial space. Then the ghost values will be recognized as convenient, though possibly nonoptimal, degrees-of-freedom associated with these ghost basis functions. The trial space is therefore

$$
V^{h, p}=\operatorname{Span}\left\{\phi_{\mathrm{j}}^{(\mathrm{p})}, \mathrm{j}=1-\mathrm{q}, \ldots, \mathrm{N}+\mathrm{q}-1\right\} .
$$

One possible disadvantage of this method, which can be easily anticipated by studying the graphs of the basis functions in Figure 1), is that the contributions to the mass matrix of the ghost values furthest from the physical domain will be extremely small for $p$ large. Thus the mass matrix will be ill-conditioned, and a rescaling of the degrees-of-freedom might be useful in practice. This effect, however, will be partially compensated by the fact that contributions to the stiffness/derivative matrices will also be small. Even still we will present numerical experiments with this method and see that it is effective in double precision arithmetic for reasonably large values of $p$ - up through $p=11$.

Extrapolation: An alternative to the ghost basis approach is to relate ghost values to values in the domain interior through an extrapolation procedure. For example, suppose $p=3$ and thus $q=2$. A 
single ghost value is needed at each boundary which can be obtained, say, by the fourth-order accurate extrapolation formulas:

$$
\begin{aligned}
u_{-1} & =4 u_{0}-6 u_{1}+4 u_{2}-u_{3}, \\
u_{N+1} & =4 u_{N}-6 u_{N-1}+4 u_{N-2}-u_{N-3},
\end{aligned}
$$

which is equivalent to modifying the basis functions $\phi_{j}^{(3)}, j=0, \ldots, 3$ and $j=N-3, \ldots, N$ :

$$
\begin{array}{ll}
\tilde{\phi}_{0}^{(3)}=\phi_{0}^{(3)}+4 \phi_{-1}^{(3)}, & \tilde{\phi}_{N}^{(3)}=\phi_{N}^{(3)}+4 \phi_{N+1}^{(3)} \\
\tilde{\phi}_{1}^{(3)}=\phi_{1}^{(3)}-6 \phi_{-1}^{(3)}, & \tilde{\phi}_{N-1}^{(3)}=\phi_{N-1}^{(3)}-6 \phi_{N+1}^{(3)}, \\
\tilde{\phi}_{2}^{(3)}=\phi_{2}^{(3)}+4 \phi_{-1}^{(3)}, & \tilde{\phi}_{N-2}^{(3)}=\phi_{N-2}^{(3)}+4 \phi_{N+1}^{(3)}, \\
\tilde{\phi}_{3}^{(3)}=\phi_{3}^{(3)}-\phi_{-1}^{(3)}, & \tilde{\phi}_{N-3}^{(3)}=\phi_{N-3}^{(3)}-\phi_{N+1}^{(3)} .
\end{array}
$$

The trial space is then

$$
\tilde{V}^{h, 3}=\operatorname{Span}\left\{\tilde{\phi}_{\mathrm{j}}^{(3)}, \mathrm{j}=0, \ldots, 3 ; \quad \phi_{\mathrm{j}}^{(\mathrm{p})}, \mathrm{j}=4, \ldots, \mathrm{N}-4 ; \quad \tilde{\phi}_{\mathrm{j}}^{(3)}, \mathrm{j}=\mathrm{N}-3, \ldots, \mathrm{N}\right\} .
$$

See Figure 2 for plots of the basis functions near the boundaries, including modifications resulting from this extrapolation procedure for $p=3$ and $p=5$.
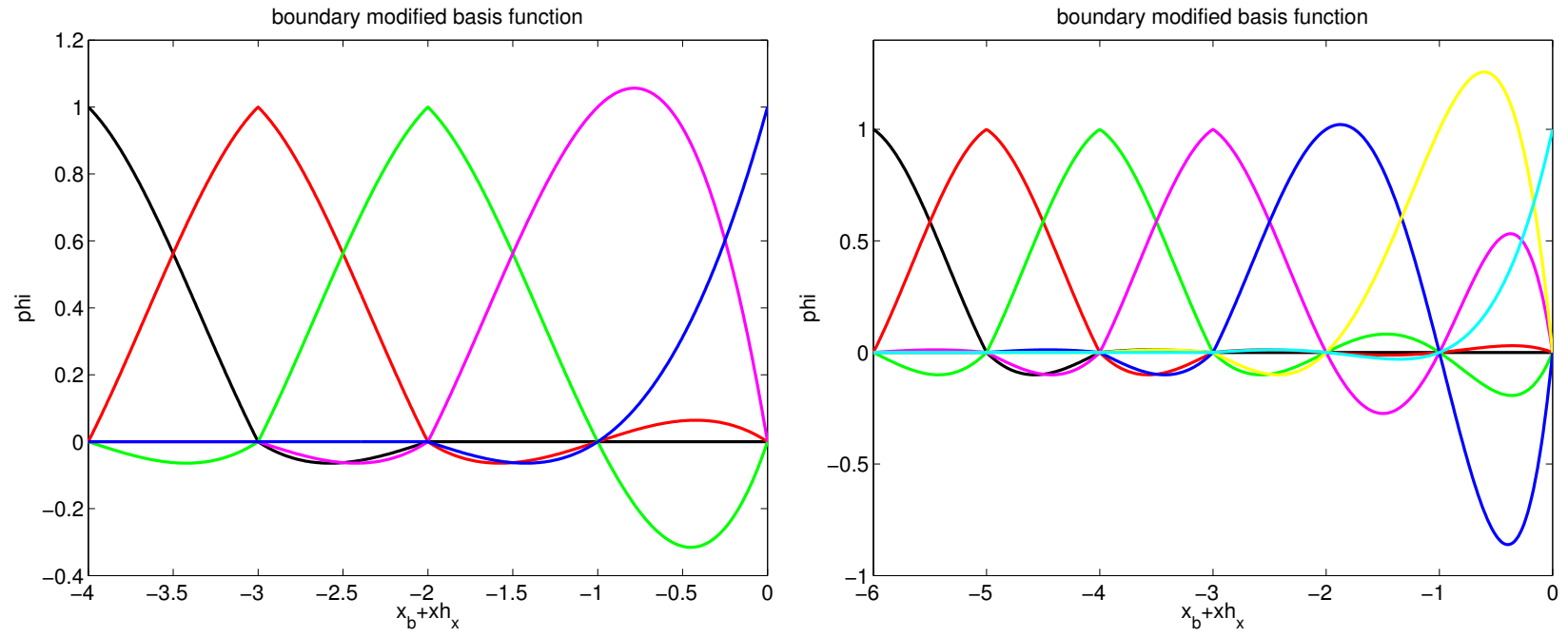

Figure 2: Basis functions near the boundary, including modifications resulting from the extrapolation closure, for $p=3$ and $p=5$. The basis function associated with each grid point are color coded, and the boundary lies at the right of the domain.

Obviously this procedure can be extended to any degree and the order of extrapolation can also be varied. In our experiments so far we have noticed some advantage of extrapolated bases over the simpler ghost basis method in terms of the spectral radius of the evolution operators at high order. However, the ghost basis may be easier to adapt to more complex applications of the Galerkin difference concept than considered here - for example their use to develop embedded boundary methods. In our experiments with Galerkin difference methods based on band-limited rather than Lagrange interpolants [16], we found it convenient to use extrapolation in concert with mass-lumping.

\subsection{Compatibility Boundary Conditions}

The above mentioned ghost basis and extrapolation boundary closures are generic in the sense that they do not directly enforce any particular boundary condition, nor are they tailored to any particular equation. 
Another approach to boundary closures uses the PDE and boundary condition to derive extra conditions which smooth solutions to the continuous problem must also satisfy, so-called compatibility boundary conditions. Such an approach has been used in the finite difference community to good effect, and we will investigate it here. For simple problems in simple domains compatibility conditions are often easy to derive. For wave equations a typical case involves either odd or even extension of functions across boundaries. As an example, consider the scalar wave equation $u_{t t}=c^{2} u_{x x}$ with the homogeneous Neumann boundary conditions $u_{x}(x=b, t)=0$. The boundary condition can be differentiated in time and combined with the governing equation to derive additional constraints on a smooth solution:

$$
\begin{gathered}
0=u_{x t t}(x=b, t)=u_{x x x}(x=b, t) \\
0=u_{x t t t t}(x=b, t)=u_{x x x x x}(x=b, t) \\
0=u_{x t t t t t t}(x=b, t)=u_{x x x x x x x}(x=b, t)
\end{gathered}
$$

These can be satisfied by insisting that the trial space be limited to functions with an even extension across $b$. In terms of ghost cells we simply have:

$$
u_{N+j}=u_{N-j} .
$$

This translates to modified basis functions

$$
\tilde{\phi}_{N-j}^{(p)}=\phi_{N-j}^{(p)}+\phi_{N+j}^{(p)}, \quad j=1, \ldots, q-1,
$$

with the trial space given by (4). Likewise for homogeneous Dirichlet conditions $u(x=b, t)=0$ we have an odd extension

which implies

$$
u_{N+j}=-u_{N-j},
$$

$$
\tilde{\phi}_{N-j}^{(p)}=\phi_{N-j}^{(p)}-\phi_{N+j}^{(p)}, \quad j=1, \ldots, q-1 .
$$

The experiments will show that this compatibility method yields superior results, and we can successfully use arbitrary values of $p$. However, the approach is difficult to generalize to more complex boundary conditions and multidimensional domains, though some successful applications in the context of standard difference approximations in mapped domains have been demonstrated [17. It may also be useful to use some small number of compatibility conditions in combination with extrapolation for the remaining unknowns in the ghost cells, although this is left to future work.

\section{Second order problems}

The construction of discrete approximations to second-order wave equations now follows a standard prescription. Assuming $c$ is constant consider

$$
u_{t t}=c^{2} u_{x x}+f(x, t),
$$

with Robin boundary conditions

$$
\begin{aligned}
u_{x}-\gamma_{a} u_{t}-\beta_{a} u & =0, \quad x=a, \\
u_{x}+\gamma_{b} u_{t}+\beta_{b} u & =0, \quad x=b,
\end{aligned}
$$

where the constants $\gamma_{a, b}, \beta_{a, b}$ are nonnegative. Then the exact solution $u$ satisfies the energy inequality

$$
\frac{d \mathcal{E}}{d t} \leq \int_{a}^{b} u_{t}(x, t) f(x, t) d x, \quad \mathcal{E}=\frac{1}{2} \int_{a}^{b} u_{t}^{2}(x, t)+c^{2} u_{x}^{2}(x, t) d x+\frac{\beta_{a}}{2} u^{2}(a)+\frac{\beta_{b}}{2} u^{2}(b) .
$$

We define a Galerkin approximation by

$$
\begin{aligned}
\int_{a}^{b} \phi_{k}^{(p)}(x) \tilde{u}_{t t}(x, t) d x= & -c^{2} \int_{a}^{b} \phi_{k, x}^{(p)} \tilde{u}_{x}(x, t) d x+\int_{a}^{b} \phi_{k}^{(p)}(x) f(x, t) d x \\
& -c^{2} \phi_{k}^{(p)}(a)\left(\gamma_{a} \tilde{u}_{t}(a, t)+\beta_{a} \tilde{u}(a, t)\right)-c^{2} \phi_{k}^{(p)}(b)\left(\gamma_{b} \tilde{u}_{t}(b, t)+\beta_{b} \tilde{u}(b, t)\right)
\end{aligned}
$$


for all $k$ with $\tilde{u}$ given by

$$
\tilde{u}(x, t)=\sum_{\alpha} u_{\alpha}(t) \phi_{\alpha}^{(p)}(x)
$$

In matrix form we have

$$
M^{(p)} U_{t t}=K^{(2, p)} U+D^{(p)} U_{t}+B^{(p)} U+F,
$$

where $U(t)$ is the vector of coefficients $u_{\alpha}, F(t)$ is the vector of inner products of $f$ with the basis functions, and the mass, $M^{(p)}$, stiffness, $K^{(2, p)}$, and boundary, $B^{(p)}, D^{(p)}$, matrices are given by

$$
\begin{aligned}
M_{k \alpha}^{(p)} & =\int_{a}^{b} \phi_{k}^{(p)}(x) \phi_{\alpha}^{(p)}(x) d x, & K_{k \alpha}^{(2, p)} & =-c^{2} \int_{a}^{b} \phi_{k, x}^{(p)}(x) \phi_{\alpha, x}^{(p)}(x) d x . \\
D_{k \alpha}^{(p)} & =-c^{2}\left(\gamma_{a} \delta_{00}+\gamma_{b} \delta_{N N}\right), & B_{k \alpha}^{(p)} & =-c^{2}\left(\beta_{a} \delta_{00}+\beta_{b} \delta_{N N}\right) .
\end{aligned}
$$

Here we have introduced the Kronecker $\delta$ associated with the nodal indices; $\delta_{00}$ is the matrix which is zero except for the single entry of 1 corresponding to the test and trial functions associated with the boundary node $x_{0}=a$, and $\delta_{N N}$ is analogously defined for the Lagrange functions of the boundary node $x_{N}=b$. Note that for simplicity we drop the tilde notation for the modified basis elements used with extrapolation or compatibility boundary conditions. Clearly the mass and stiffness matrices will be banded with bandwidth $2 p+1$, the same as for degree $p$ continuous FE. Thus the method can be viewed as a particular compact difference scheme.

To handle a homogeneous Dirichlet condition $u=0$ at a boundary we simply remove the basis element whose degree of freedom is the function value at the boundary. That is, we take the subspace, $V_{0}^{h, p}$, of $V^{h, p}$ of functions vanishing at Dirichlet boundaries, and in terms of the mass and stiffness matrices defined above remove the rows and columns with indices 0 and $N$. In this case, and the case of Neumann boundary conditions, $D^{(p)}=B^{(p)}=0$. In all cases we have a discrete energy identity analogous to 11

$$
\frac{d}{d t}\left(\frac{1}{2} U_{t}^{T} M^{(p)} U_{t}+\frac{1}{2} U^{T} K^{(p)} U+\frac{\beta_{a}}{2} u_{0}^{2}+\frac{\beta_{b}}{2} u_{N}^{2}\right) \leq U_{t}^{T} F .
$$

\subsection{Convergence}

As the proposed Galerkin Difference method is, in fact, a standard Galerkin approximation the convergence of the method follows directly from the well-known Galerkin theory combined with the approximation properties of our basis. Here we consider the semidiscrete problem; that is we assume that (14) is solved exactly in time. Then we have the following Theorem, adapted from [18 with specialization to this simple case, proving that our method converges at order $p+1$.

Theorem 1 (Baker 18). Suppose the solution, $u(x, t)$, to (8) is sufficiently smooth, and the discrete initial data, $\tilde{u}(x, 0), \frac{\partial \tilde{u}}{\partial t}(x, 0)$ is given by the $L^{2}$-projection of $u(x, 0)$ and $\frac{\partial u}{\partial t}(x, 0)$ onto $V^{h, p}$, for boundary conditions (9) and 10), or $V_{0}^{h, p}$ in the case of Dirichlet conditions. Then for any $T>0$ there exists $C(u, T)$ such that

$$
\|u(\cdot, T)-\tilde{u}(\cdot, T)\|_{L^{2}(a, b)} \leq C(u, T) h^{p+1} .
$$

Proof: We need only check that for $1 \leq s \leq p+1$

$$
\inf _{\tilde{v} \in V^{(h, p)}}\left(\|v-\tilde{v}\|_{L^{2}(a, b)}+h\|v-\tilde{v}\|_{H^{1}(a, b)}\right) \leq h^{s}\|v\|_{H^{s}(a, b)},
$$

which follows from well-known facts about piecewise polynomial approximation.»

Remark 1. In practice we simply interpolate the initial data rather than use the projections required by Baker's Theorem. As will be seen below, convergence at the design order is still observed. We also note that the result applies for problems with variable coefficients and in multiple space dimensions.

Remark 2. An alternative error analysis based on finite-difference methodology is also possible. With energy stability established using Galerkin methods we can leverage the fact that the compact difference operators we construct are accurate to order $p+1$. 


\begin{tabular}{|c|c|c|c|c|c|c|c|}
\hline$\alpha$ & -3 & -2 & -1 & 0 & 1 & 2 & 3 \\
\hline \hline$M_{\alpha}^{(3)}$ & $\frac{31 h}{15120}$ & $\frac{-3 h}{70}$ & $\frac{257 h}{1680}$ & $\frac{733 h}{945}$ & $\frac{257 h}{1680}$ & $\frac{-3 h}{70}$ & $\frac{31 h}{15120}$ \\
\hline$K_{\alpha}^{(2,3)}$ & $\frac{-7}{360 h}$ & $\frac{1}{30 h}$ & $\frac{25}{24 h}$ & $\frac{-19}{9 h}$ & $\frac{25}{24 h}$ & $\frac{1}{30 h}$ & $\frac{-7}{360 h}$ \\
\hline
\end{tabular}

Table 1: Coefficients of the mass and stiffness matrices for interior points using $p=3$.

\subsection{Dispersion analysis}

On a uniform grid in the interior of the domain the matrices $M^{(p)}$ and $K^{(2, p)}$ are translation invariant and thus amenable to Fourier analysis. For example Table 1 gives the coefficients for the mass and stiffness matrices for $p=3$ for all points sufficiently far from boundaries. Similarly, Table 2 gives the coefficients for the mass and stiffness matrices for $p=5$. Note that the symmetries $M_{\alpha}^{(p)}=M_{-\alpha}^{(p)}$ and $K_{\alpha}^{(2, p)}=K_{-\alpha}^{(2, p)}$, have been used to save space in the presentation.

\begin{tabular}{|c|c|c|c|c|c|c|}
\hline$\alpha$ & 0 & 1 & 2 & 3 & 4 & 5 \\
\hline \hline$M_{\alpha}^{(5)}$ & $\frac{455963 h}{554400}$ & $\frac{906919 h}{6652800}$ & $\frac{-12421 h}{207900}$ & $\frac{59053 h}{4435200}$ & $\frac{-3937 h}{3326400}$ & $\frac{313 h}{4435200}$ \\
\hline$K_{\alpha}^{(2,5)}$ & $\frac{-12053}{5400 h}$ & $\frac{529}{480 h}$ & $\frac{8}{135 h}$ & $\frac{-3389}{60480 h}$ & $\frac{517}{45360 h}$ & $\frac{-89}{129600 h}$ \\
\hline
\end{tabular}

Table 2: Coefficients of the mass and stiffness matrices for interior points using $p=5$.

Fourier analysis can be used to derive the symbol of the Galerkin difference approximation to the second derivative as

$$
-k^{2} \approx \frac{\sum_{\alpha=-p}^{p} K_{\alpha}^{(2, p)} \exp (i \alpha \eta)}{\sum_{\alpha=-p}^{p} M_{\alpha}^{(p)} \exp (i \alpha \eta)} \equiv \frac{1}{h^{2}} \mathcal{S}^{(2, p)}(\eta)
$$

where $k$ is the physical wave number and $\eta=k h$ is the normalized grid wave number. Note that the number of discrete points per wavelength (PPW) is $P P W=2 \pi / \eta$, which will be used in discussing both the dispersion error and the numerical results to follow. The dispersion properties of the methods can be assessed by investigating the error of the approximations in (20), and later for the first-order system in 45), for small $\eta$ which corresponds to small $h$ and finite wave number $k$. For small $\eta$ the approximation is expressed as a polynomial in powers of $\eta$, exposing the local error of the discrete operator. For $p=3$ we find

$$
\mathcal{S}^{(2,3)}(\eta)=-\eta^{2}-\frac{17}{6048} \eta^{8}+\ldots
$$

and subsequently

$$
-k^{2} \approx-k^{2}-\frac{17}{6048} k^{8} h^{6}
$$

Therefore the dispersion error for the case $p=3$ converges at 6 th order. Given the error analysis above in Section 4.1, which predicts 4 th order convergence, we recognize this as a superconvergence phenomenon. This is a well-known effect for Galerkin methods; see, e.g., [19] and references therein. The practical consequence is that, depending on the problem and the resolution required, we may observe much more rapid convergence than predicted by Theorem 1. This will be detailed in the experiments below.

For $p=5$ the leading terms are in the expansion of the Fourier symbols for small $\eta$ are

$$
\mathcal{S}^{(2,5)}(\eta)=-\eta^{2}-\frac{4121}{34214400} \eta^{12}+\ldots
$$

and therefore the dispersion error using $p=5$ is 10 th order.

The procedure for using any order polynomial reconstruction to produce a discretization is straightforward. However, the coefficients involved in the discretization are unwieldy and so we choose not to present 
them here. We do however present the leading order dispersion error terms. For $p=7$ and $p=9$ the expansion of the Fourier symbols for small $\eta$ is

$$
\begin{aligned}
& \mathcal{S}^{(2,7)}(\eta)=-\eta^{2}-\frac{14981203}{2615348736000} \eta^{16}+\ldots \\
& \mathcal{S}^{(2,9)}(\eta)=-\eta^{2}-\frac{44734915633}{154821036883968000} \eta^{20}+\ldots
\end{aligned}
$$

These expansions imply that the interior discretization using $p=7$ has 14 th order dispersion error, while the interior discretization using $p=9$ has 18 th order dispersion error.

It is useful to plot the behavior of the discrete Fourier symbols for the various orders as a function of normalized wave number $\eta$. Such plots, along with error plots, are presented in Figure 3 . The dotted line in the second plot represents an error tolerance of $10^{-4}$ and is included in the plot for convenient reference. Note that the axes in these graphs can be reinterpreted in terms of grid points per wavelength, $P P W=2 \pi / \eta$. Thus we see that to obtain an error of $10^{-4}$ for $p=3$ one would need roughly $12 \mathrm{PPW}$, while for $p=9$ only $4 \mathrm{PPW}$ would be needed. Note also that the imaginary part of $\mathcal{S}^{(2)}$ is exactly zero.
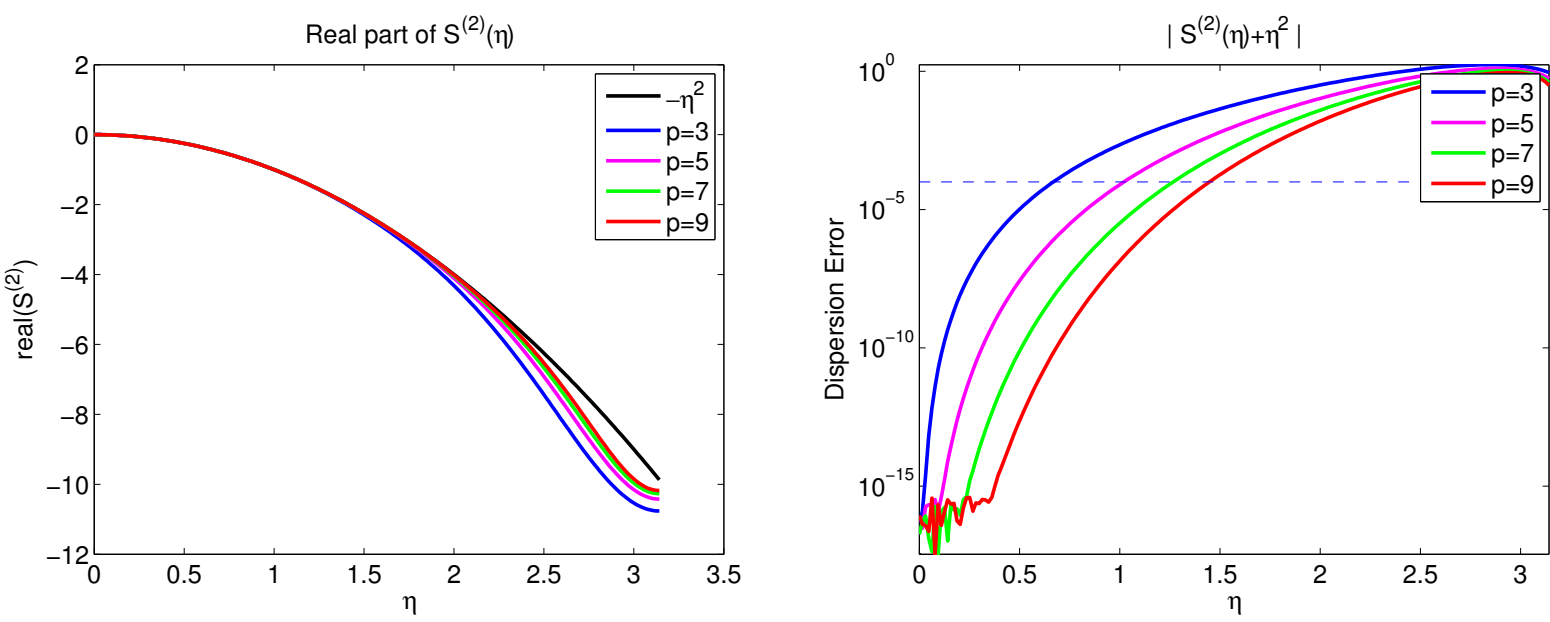

Figure 3: Symbols of the second order difference operators for various orders. Note we show the curves only for positive wave number as the negative wave numbers are determined via even symmetry.

\subsection{Spectrum of the difference operators}

The maximum size of the symbol is an important quantity relating to the stiffness of the difference operator. For the interior discretization analytic expressions can be found and Table 3 reports the maximum to 4 significant figures. Importantly, the spectrum is seen to decay toward $\pi^{2}$, the spectrum of the continuous

\begin{tabular}{|c||c|c|c|c|}
\hline$p$ & 3 & 5 & 7 & 9 \\
\hline \hline $\max \mid \mathcal{S}^{(2, p)}$ & 10.76 & 10.42 & 10.27 & 10.18 \\
\hline
\end{tabular}

Table 3: Table of maximum eigenvalues for the interior approximation to the second derivatives for various orders.

operator, as $p$ increases. Thus there is very little stiffness introduced by the discretization process which is not already present in the continuous operator.

In the present case of the second-order wave equation the time step restriction for an explicit method will be proportional to the square root of the maximum eigenvalue, and the ideal upper bound for this maximal eigenvalue is $\frac{\pi^{2}}{h^{2}}$. Note that Table 3 shows that the maximum eigenvalues for the interior schemes 
alone are slightly larger than $\frac{\pi^{2}}{h^{2}} \approx \frac{9.87}{h^{2}}$. Thus for the periodic case the maximal time step is at least $95 \%$ if the ideal value. To determine the effect of the generic boundary closures on the time step we compute the maximum eigenvalues for the GD discretization, including boundary closures, on a domain with 200 points. We define a time-stepping efficiency factor, $E$, by dividing the square root of the ideal maximal eigenvalue by the square root of the computed maximum eigenvalue. This normalized results approximately represent the loss in efficiency for explicit time stepping attributable to the boundary closures since the discretization on a periodic domain shows very little degradation in the maximal stable time step. The first observation we make is that for the case of compatibility conditions there is no growth of the eigenvalues relative to the periodic case. The results for the ghost basis and extrapolation methods are reported in Table 4 . There it can be seen that for generic boundary closures the loss of efficiency is slightly greater for Neumann rather than Dirichlet conditions, and the efficiency loss increases with degree $p$. Furthermore, the extrapolation basis results are superior in this measure. However, even for the ghost basis we view the growth of the eigenvalues as moderate - for $p=9$ we have $E=0.382$ for the Dirichlet problem and $E=0.225$ for the Neumann problem.

\begin{tabular}{|c||c|c|c|c|}
\hline$p$ & 3 & 5 & 7 & 9 \\
\hline \hline$h^{2} \rho\left(\left(M_{D}^{(p, G)}\right)^{-1} K_{D}^{(2, p, G)}\right)$ & 16.79 & 56.33 & 62.69 & 67.81 \\
\hline$E_{D}^{G}$ & 0.767 & 0.419 & 0.397 & 0.382 \\
\hline \hline$h^{2} \rho\left(\left(M_{D}^{(p, X)}\right)^{-1} K_{D}^{(2, p, X)}\right)$ & 10.76 & 10.42 & 11.68 & 16.17 \\
\hline$E_{D}^{X}$ & 0.958 & 0.973 & 0.919 & 0.781 \\
\hline \hline$h^{2} \rho\left(\left(M_{N}^{(p, G)}\right)^{-1} K_{N}^{(2, p, G)}\right)$ & 49.85 & 66.96 & 158.96 & 194.42 \\
\hline$E_{N}^{G}$ & 0.445 & 0.384 & 0.249 & 0.225 \\
\hline \hline$h^{2} \rho\left(\left(M_{N}^{(p, X)}\right)^{-1} K_{N}^{(2, p, X)}\right)$ & 15.04 & 25.67 & 40.85 & 60.17 \\
\hline$E_{N}^{X}$ & 0.810 & 0.620 & 0.492 & 0.405 \\
\hline
\end{tabular}

Table 4: Table of maximum eigenvalues and normalized stability constraint for second derivatives with Dirichlet and Neumann boundary conditions for the ghost basis and extrapolation basis closures at various orders. Here the subscript $D$ denotes Dirichlet conditions and $N$ Neumann conditions while the superscripts $G$ and $X$ denote, respectively, the ghost basis and extrapolation basis closures.

\subsection{Numerical experiments}

To evaluate the accuracy of the Galerkin difference operators in a concrete setting, and in particular to observe any potential superconvergence results relating to the higher order dispersion errors discussed in Section 4.2. we study convergence with grid refinement when using the GD methods to compute the solution to the wave equation $u_{t t}=u_{x x}$ with exact solutions of the form

$$
u(x, t)=\sin \left(\frac{m}{2} \pi x\right) \cdot \cos \left(\frac{m}{2} \pi t\right),
$$

for $x \in(0,1), t \in\left(0, t_{f}\right), m$ is an odd integer picked for a particular test case, and we impose homogeneous Dirichlet boundary conditions at $x=0$ and Neumann conditions at $x=1$. Note that for the presentation of results we choose the final time to be either $t_{f}=1.5$ or $t_{f}=100.5$ which do not correspond to times where the solution or its time derivative are zero since this can complicate the interpretation of the results. Time stepping is performed using a Runge-Kutta-Taylor method of order $2 p+1$ with $\Delta t / h=1 / 4$. This very high-order time integrator is chosen to be certain that any superconvergence results associated with the accurate dispersion relation will be observed.

As a first test consider $m=41$ and $t_{f}=100.5$. This case is meant to mimic a physical scenario where the domain contains multiple wavelengths in space, 10.25 for this case, and the final time is sufficiently late that disturbances have the opportunity to traverse the domain many times, here 100.5 times. Such a scenario is 
common for wave propagation problems. Figure 4 shows results of the convergence studies using the $L^{2}$-norm for all three boundary closures and $p=1,3,5,9$. The mesh refinement is based on increasing the number of discretization points $n$ as $n=180: 20: 460$ for $p=3, n=60: 20: 340$ for $p=5, n=50: 15: 260$ for $p=7$, and $n=40: 15: 250$ for $p=9$. In the figure there is one plot for each $p$ considered, and each plot includes reference lines whose slope corresponds to convergence at the rate $O\left(h^{p+1}\right)$, and $O\left(h^{2 p}\right)$, the convergence and superconvergence rates indicated in Sections 4.1 and 4.2 respectively.
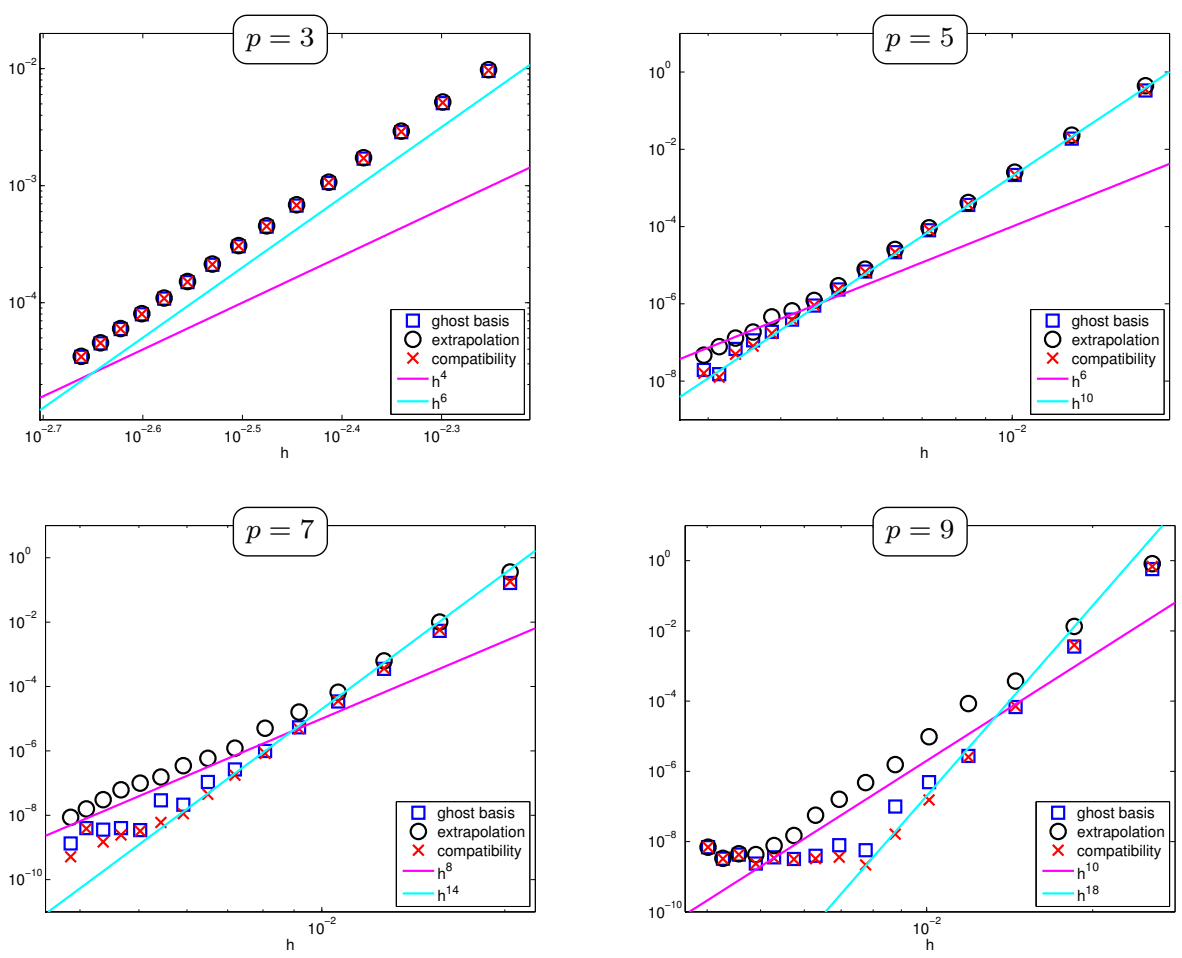

Figure 4: Results for convergence studies using the $L^{2}$-norm for the three boundary closures and $p=3,5,7,9$. Here $m=41$ and $t_{f}=100.5$, corresponding to 10.25 wavelengths in space and 100.5 periods in time.

The plots in Figure 4 illustrate that all three methods attain average convergence rates which are much higher than $O\left(h^{p+1}\right)$, and in fact for many cases the superconvergent rate $O\left(h^{2 p}\right)$ is convincingly demonstrated. In all cases saturation of the error within the confines of double precision arithmetic is apparently occurring near $10^{-9}$ which is not entirely surprising given the large number of time steps used to arrive at the final time. Also in the plots one can see that as the error becomes smaller, and especially for higher $p$, the extrapolation closure, and to a lesser extent the ghost basis closure, are falling off the superconvergent rate and tending instead toward convergence at $O\left(h^{p+1}\right)$ as predicted by Theorem 1 .

Through our experimentation we have found that convergence at rates higher than the prediction in Theorem 1 tends to occur when the errors is above a threshold which depends on the particulars of the test case. As a general rule this threshold is found to decrease as both the spatial and temporal domains increase in size. To illustrate this phenomenon consider the case with $m=7$ and $t_{f}=1.5$. Figure 5 shows results from convergence studies using $n=10: 5: 300$ for $p=3$ and $n=10: 2: 40$ for $p=9$. From these plots one can notice that both the ghost basis closure and the extrapolation closure do in fact tend toward convergence at the rate $O\left(h^{p+1}\right)$, while the closure based on compatibility retains its superconvergent rate of $O\left(h^{2 p}\right)$ for this case. Note also that the saturation of the error within the confines of double precision occurs here at the much lower value of $10^{-14}$ which we attribute to the smaller wave number and integration time.

We now investigate the limits of the superconvergent behavior when compatibility boundary conditions are used by running a series of convergence test for extremely high values of $p$. Here we choose $m=175$, corresponding to 43.75 wavelengths, and use $t=1.5$. Tests are run using $p=11,41,71$, and 101 and the 

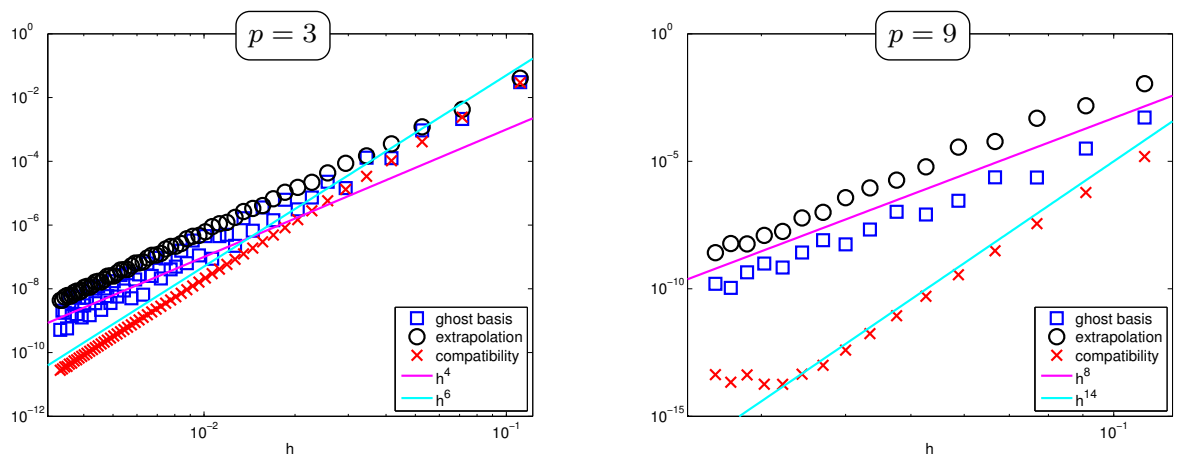

Figure 5: Results for convergence studies using the $L^{2}$-norm for the three boundary closures and $p=3,9$. Here $m=7$ and $t_{f}=1.5$, corresponding to 1.75 wavelengths in space and 1.5 periods in time.

number of spatial grid points for all cases is $n=100: 2: 175$. Figure 6 shows convergence results for this test case. The left plot in the figure includes reference lines indicating the superconvergence rate $O\left(h^{2 p}\right)$ for the various orders of $p$. The right plot in the figure includes reference lines indicating the rates $O\left(h^{18}\right)$, $O\left(h^{60}\right), O\left(h^{95}\right), O\left(h^{130}\right)$, which are approximations of the measured rates of convergence for the various schemes. From the plots one can notice that the convergence rates for the four simulations appear to be tending toward the superconvergent rate, but, particularly for the higher order methods, the limits of double precision do not permit enough grid refinement to achieve the full $2 p$ rate. Note that in this case the coarsest grid in this study corresponds to just $2.3 \mathrm{PPW}$ while the finest has $4 \mathrm{PPW}$. The method with $p=101$ saturates within double precision at just $2.9 \mathrm{PPW}$, for $p=75$ saturation occurs at around $3.2 \mathrm{PPW}$, and for $p=41$ saturation is near the finest $4 \mathrm{PPW}$.
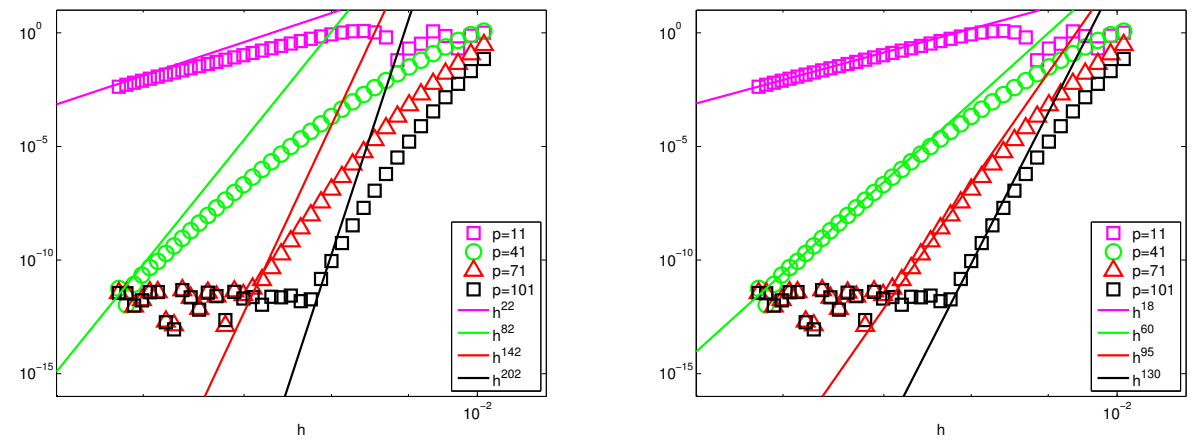

Figure 6: Results for convergence studies using the $L^{2}$-norm for the compatibility boundary closures and $p=11,41,71$, and 101. Here $m=175$ and $t_{f}=1.5$, corresponding to 43.75 wavelengths in space and 1.5 periods in time. At left the reference lines indicate the superconvergence rate $O\left(h^{2 p}\right)$. At right the reference lines indicate the rates $O\left(h^{18}\right)$, $O\left(h^{60}\right), O\left(h^{95}\right), O\left(h^{130}\right)$ which approximate the measured rates for the various schemes.

It is natural to ask if a method with accuracy $2 p$ (or $2 p+2$ for first order systems) can be obtained by altering the basis in a neighborhood of the boundaries. For $p=1$ it is shown by Gottlieb and coworkers in [20] that this cannot be done in a straightforward way. We have not investigated thoroughly what possibilities exist to bypass their analysis. 


\section{First order problems}

We now consider first order symmetric systems of the form

$$
u_{t}=A u_{x}+f(x, t)
$$

where $u(x, t) \in \mathbb{R}^{n}$, and $A=A^{T}$. We assume, without loss of generality, that $A$ is in block diagonal form:

$$
A=\left(\begin{array}{cc}
A^{+} & 0 \\
0 & -A^{-}
\end{array}\right)
$$

with $A^{ \pm} \geq 0$, i.e. positive semidefinite, and partition $u$ accordingly. Lastly we suppose the boundary conditions take the form

$$
\begin{aligned}
u^{-}=R_{a} u^{+}+g_{a}(t), & x=a, \\
u^{+}=R_{b} u^{-}+g_{b}(t), & x=b,
\end{aligned}
$$

with

$$
\begin{aligned}
& A^{+}-R_{a}^{T} A^{-} R_{a} \geq 0 \\
& A^{-}-R_{b}^{T} A^{+} R_{b} \geq 0 .
\end{aligned}
$$

With these assumptions one deduces the basic energy inequality for the homogeneous problem with $f=0$, $g_{a, b}=0$

$$
\frac{d\|u(\cdot, t)\|_{L^{2}}^{2}}{d t} \leq 0 .
$$

We construct discrete approximations to $(22)$ using the framework from [21. Approximating $u$ in the space $\left(V^{h, p}\right)^{n}$, that is setting

$$
\tilde{u}_{i}(x, t)=\sum_{\alpha} u_{i, \alpha}(t) \phi_{\alpha}^{(p)}(x)
$$

we impose for all $(i, k)$

$$
\begin{aligned}
\int_{a}^{b} \phi_{k}^{(p)}(x) \tilde{u}_{i, t}(x, t) d x= & \int_{a}^{b} \phi_{k}^{(p)}(x)\left(\sum_{j} a_{i j} \tilde{u}_{j, x}(x, t)+f_{i}(x, t)\right) d x \\
& +\phi_{k}^{(p)}(b)\left(\sum_{j} a_{i j}\left(u_{b, j}^{*}(t)-\tilde{u}_{j}(b, t)\right)\right)-\phi_{k}^{(p)}(a)\left(\sum_{j} a_{i j}\left(u_{a, j}^{*}(t)-\tilde{u}_{j}(a, t)\right)\right) .
\end{aligned}
$$

Here the vectors $u_{a}^{*}, u_{b}^{*}$ are chosen to impose fluxes consistent with the boundary conditions (24)-(25). Many choices are possible, but in our experiments we opt for upwind fluxes defined by:

$$
\begin{aligned}
& u_{a}^{*,+}(t)=\tilde{u}^{+}(a, t), \quad u_{a}^{*,-}(t)=R_{a} u_{a}^{*,+}(t)+g_{a}(t), \\
& u_{b}^{*,-}(t)=\tilde{u}^{-}(b, t), \quad u_{b}^{*,+}(t)=R_{b} u_{b}^{*,-}(t)+g_{b}(t) .
\end{aligned}
$$

In matrix form we then have

$$
I \otimes M^{(p)} U_{t}=A \otimes K^{(1, p)} U+\Gamma_{N} \otimes \delta_{N N} U+\left(\begin{array}{c}
A^{+} g_{b}(t) \\
0
\end{array}\right) \otimes E_{N}+\Gamma_{0} \otimes \delta_{00} U+\left(\begin{array}{c}
0 \\
A^{-} g_{a}(t)
\end{array}\right) \otimes E_{0}+F,
$$

where $M^{(p)}$ is the mass matrix defined in 15 and the other matrices are given by

$$
K_{k \alpha}^{(1, p)}=\int_{a}^{b} \phi_{k}^{(p)}(x) \phi_{\alpha, x}^{(p)}(x) d x, \quad \Gamma_{N}=\left(\begin{array}{cc}
-A^{+} & A^{+} R_{b} \\
0 & 0
\end{array}\right), \quad \Gamma_{0}=\left(\begin{array}{cc}
0 & 0 \\
A^{-} R_{a} & -A^{-}
\end{array}\right) .
$$


Here $E_{0}$ and $E_{N}$ denote the columns of the identity matrix associated with the Lagrange function of the boundary nodes, $x_{0}=a, x_{N}=b$. That is, vectors all of whose entries are 0 except for 1 in positions 0 and $N$ respectively.

With these choices and homogeneous data it is straightforward to prove an energy estimate. Using (31)- 32 we have

$$
\begin{aligned}
\frac{d\|\tilde{u}(\cdot, t)\|_{L^{2}}^{2}}{d t}= & \tilde{u}^{T}(b, t) A\left(2 u_{b}^{*}(t)-\tilde{u}(b, t)\right)-\tilde{u}^{T}(a, t) A\left(2 u_{a}^{*}(t)-\tilde{u}(a, t)\right) \\
= & \tilde{u}^{+, T}(b, t) A^{+}\left(2 u_{b}^{*,+}(t)-\tilde{u}^{+}(b, t)\right)-\tilde{u}^{-, T}(b, t) A^{-}\left(2 u_{b}^{*,-}(t)-\tilde{u}^{-}(b, t)\right) \\
& -\tilde{u}^{+, T}(a, t) A^{+}\left(2 u_{a}^{*,+}(t)-\tilde{u}^{+}(a, t)\right)+\tilde{u}^{-, T}(a, t) A^{-}\left(2 u_{a}^{*,-}(t)-\tilde{u}^{-}(a, t)\right) \\
= & u_{b}^{*,+, T} A^{+} u_{b}^{*,+}-u_{b}^{*,-, T} A^{-} u_{b}^{*,-}-\left(\tilde{u}^{+}(b, t)-u_{b}^{*,+}\right)^{T} A^{+}\left(\tilde{u}^{+}(b, t)-u_{b}^{*,+}\right) \\
& -u_{a}^{*,+, T} A^{+} u_{a}^{*,+}+u_{a}^{*,-, T} A^{-} u_{a}^{*,-}-\left(\tilde{u}^{-}(a, t)-u_{a}^{*,-}\right)^{T} A^{-}\left(\tilde{u}^{-}(a, t)-u_{a}^{*,-}\right) \\
= & -u_{b}^{*,-, T}\left(A^{-}-R_{b}^{T} A^{+} R_{b}\right) u_{b}^{*,-}-u_{a}^{*,+, T}\left(A^{+}-R_{a}^{T} A^{-} R_{a}\right) u_{a}^{*,+} \\
& -\left(\tilde{u}^{+}(b, t)-u_{b}^{*,+}\right)^{T} A^{+}\left(\tilde{u}^{+}(b, t)-u_{b}^{*,+}\right)-\left(\tilde{u}^{-}(a, t)-u_{a}^{*,-}\right)^{T} A^{-}\left(\tilde{u}^{-}(a, t)-u_{a}^{*,-}\right) \\
\leq & 0 .
\end{aligned}
$$

Note that with our upwind flux there is additional dissipation proportional to the jumps in the incoming variables.

\subsection{Convergence}

To derive optimal error estimates for smooth solutions we must define an appropriate comparison function from $\left(V^{h, p}\right)^{n}$, typically the so-called upwind projection 22. Although it is straightforward to define such a projection in our setting, we have not yet established the necessary existence and approximation properties. Thus we will settle for a straightforward adaptation of the arguments in [21] to establish convergence at a slightly suboptimal rate. Optimal convergence as well as superconvergence at coarse resolutions will be observed in the numerical experiments.

Let $\tilde{v}$ be the $L^{2}$-projection of $u$ into $\left(V^{h, p}\right)^{n}$. For $\delta=u-\tilde{v}$ and a sufficiently smooth solution we have, for constants $C$ independent of $h$ :

$$
\begin{gathered}
\int_{a}^{b} \delta_{t} \cdot \psi=0, \quad \forall \psi \in\left(V^{h, p}\right)^{n} \\
\|\delta\|_{L^{\infty}[a, b]} \leq C h^{p+1} \\
\|\delta\|_{L^{2}(a, b)} \leq C h^{p+1} \\
\left\|\delta_{x}\right\|_{L^{2}(a, b)} \leq C h^{p} .
\end{gathered}
$$

In addition we note the standard polynomial inverse inequalities (e.g. [23, Thm. 3.2.6]) imply for all $v \in\left(V^{h, p}\right)^{n}:$

$$
|v(a)|+|v(b)| \leq C h^{-1 / 2}\|v\|_{L^{2}(a, b)} .
$$

(Here and in the following we use $|\cdot|$ to denote the Euclidean norm in $\mathbb{R}^{n}$.)

Using these facts we can prove the following Theorem. Note that this is one half order worse than the corresponding result in 21] due to the fact that $\delta$ is not orthogonal to derivatives of functions in $\left(V^{h, p}\right)^{n}$, as it would be for polynomial approximations.

Theorem 2. Suppose the solution, $u(x, t)$, to (22) satisfying the boundary conditions (24)-(25) is sufficiently smooth, and the discrete initial data, $\tilde{u}(x, 0)$, is given by the $L^{2}$-projection of $u(x, 0)$ onto $\left(\bar{V}^{h, p}\right)^{n}$. Then for any $T>0$ here exists $C(u, p)$ such that

$$
\|u(\cdot, T)-\tilde{u}(\cdot, T)\|_{L^{2}(a, b)} \leq C(u, p)\left(T h^{p}+h^{p+1}\right) .
$$


Proof: Let $e=u-\tilde{u}=\delta+\tilde{e}, \tilde{e}=\tilde{v}-\tilde{u}$. Noting that:

$$
\int_{a}^{b} \tilde{e} \cdot e_{t}=\int_{a}^{b} \tilde{e}^{T} A e_{x}+\tilde{e}^{T}(b, t) A\left(e_{b}^{*}(t)-e(b, t)\right)-\tilde{e}^{T}(a, t) A\left(e_{a}^{*}(t)-e(a, t)\right),
$$

Substituting for $e$, using the energy inequality (35), the definition of the starred states, the approximation results (36)-39), and the inverse inequality applied to $\tilde{e}$, we have

$$
\begin{aligned}
\frac{1}{2} \frac{d\|\tilde{e}\|_{L^{2}(a, b)}^{2}}{d t}= & \int_{a}^{b} \tilde{e}^{T} A \tilde{e}_{x}+\tilde{e}^{+, T}(b, t) A^{+}\left(\tilde{e}_{b}^{*,+}(t)-\tilde{e}^{+}(b, t)\right)+\tilde{e}^{-, T}(a, t) A^{-}\left(\tilde{e}_{a}^{*,-}(t)-\tilde{e}^{-}(a, t)\right) \\
& +\int_{a}^{b} \tilde{e}^{T}\left(A \delta_{x}-\delta_{t}\right)+\tilde{e}^{+, T}(b, t) A^{+}\left(\delta_{b}^{*,+}(t)-\delta^{+}(b, t)\right)+\tilde{e}^{-, T}(a, t) A^{-}\left(\delta_{a}^{*,-}(t)-\delta^{-}(a, t)\right) \\
\leq & C h^{p}\|\tilde{e}\|_{L^{2}(a, b)}+C h^{p+1}\left(\left|\tilde{e}^{-}(a, t)\right|+\left|\tilde{e}^{+}(b, t)\right|\right) \\
\leq & C h^{p}\|\tilde{e}\|_{L^{2}(a, b)} .
\end{aligned}
$$

Since $\tilde{e}(x, 0)=0$ a direct integration of 430 implies

$$
\|\tilde{e}\|_{L^{2}(a, b)} \leq C T h^{p} .
$$

The final result follows from $(38)$ and the triangle inequality. $\diamond$

\subsection{Dispersion analysis}

As in the second order case discussed above, for uniform grids the translation invariance of the matrices $M^{(p)}$ and $K^{(1, p)}$ in the interior imply that they are amenable to Fourier analysis. We then have

$$
i k \approx \frac{\sum_{\alpha=-p}^{p} K_{\alpha}^{(1, p)} \exp (i \alpha \eta)}{\sum_{\alpha=-p}^{p} M_{\alpha}^{(p)} \exp (i \alpha \eta)} \equiv \frac{1}{h} \mathcal{S}^{(1, p)}(\eta)
$$

where as before $k$ is the wave number, $\eta=k h$ and $\mathcal{S}^{(1, p)}(\eta)$ is the Fourier symbol for the first-order operator built using $p$ th order reconstruction. In analogy with Table 1. Table 5 gives the coefficients for the stiffness matrix $K^{(1,3)}$. Similarly Table 6 gives the coefficients for $K^{(1,5)}$. Note that the symmetry $K_{\alpha}^{(1, p)}=-K_{-\alpha}^{(1, p)}$

\begin{tabular}{|c|c|c|c|c|c|c|c|}
\hline$\alpha$ & -3 & -2 & -1 & 0 & 1 & 2 & 3 \\
\hline \hline$K_{\alpha}^{(1,3)}$ & $\frac{-1}{720}$ & $\frac{4}{45}$ & $\frac{-97}{144}$ & 0 & $\frac{97}{144}$ & $\frac{-4}{45}$ & $\frac{1}{720}$ \\
\hline
\end{tabular}

Table 5: Coefficients of the first order stiffness matrix for interior points using $p=3$.

has been used to save space.

\begin{tabular}{|c|c|c|c|c|c|c|}
\hline$\alpha$ & 0 & 1 & 2 & 3 & 4 & 5 \\
\hline \hline$K_{\alpha}^{(1,5)}$ & 0 & $\frac{7267}{9600}$ & $\frac{-23761}{151200}$ & $\frac{1147}{57600}$ & $\frac{-83}{129600}$ & $\frac{103}{3628800}$ \\
\hline
\end{tabular}

Table 6: Coefficients of the first order stiffness matrix for interior points using $p=5$.

To investigate the local error, the Fourier symbol is expanded in Taylor series around $\eta=0$. For $p=3$ we have

$$
\mathcal{S}^{(1,3)}(\eta)=i \eta-i \frac{103}{453600} \eta^{9}+\ldots
$$

where only the leading term has been retained for brevity. Substituting back into 45 we find

$$
i k \approx i k-i \frac{103}{453600} k^{9} h^{8} .
$$


Therefore the dispersion error for the case $p=3$ converges to 8th order, double the expected order for degree three approximations. Similarly for $p=5$ the leading terms are in the expansion of the Fourier symbols for small $\eta$ are

$$
\mathcal{S}^{(1,5)}(\eta)=i \eta-i \frac{379397}{36324288000} \eta^{13}+\ldots
$$

corresponding to 12 th order accuracy.

For larger values of $p$ the leading order error terms follow the same pattern. For $p=7$ and $p=9$ the expansions of the Forurier symbols for small $\eta$ are

$$
\begin{aligned}
& \mathcal{S}^{(1,7)}(\eta)=i \eta-i \frac{1964407}{3810936729600} \eta^{17}+\ldots \\
& \mathcal{S}^{(1,9)}(\eta)=i \eta-i \frac{22424299416863}{843000545833205760000} \eta^{21}+\ldots
\end{aligned}
$$

These expansions imply that the interior discretization is accurate to order $2 p+2$ for these cases.

Plots of the discrete Fourier symbols for the various orders as a function of wave number $\eta$ are presented in Table 7. with the exact Fourier symbol also plotted for comparison. Note that the real part of $\mathcal{S}^{(1)}$ is exactly zero. We also plot the dispersion error, with the dotted line representing an error tolerance of $10^{-4}$. The axes in these graphs can be reinterpreted in terms of grid points per wavelength, $P P W=2 \pi / \eta$.
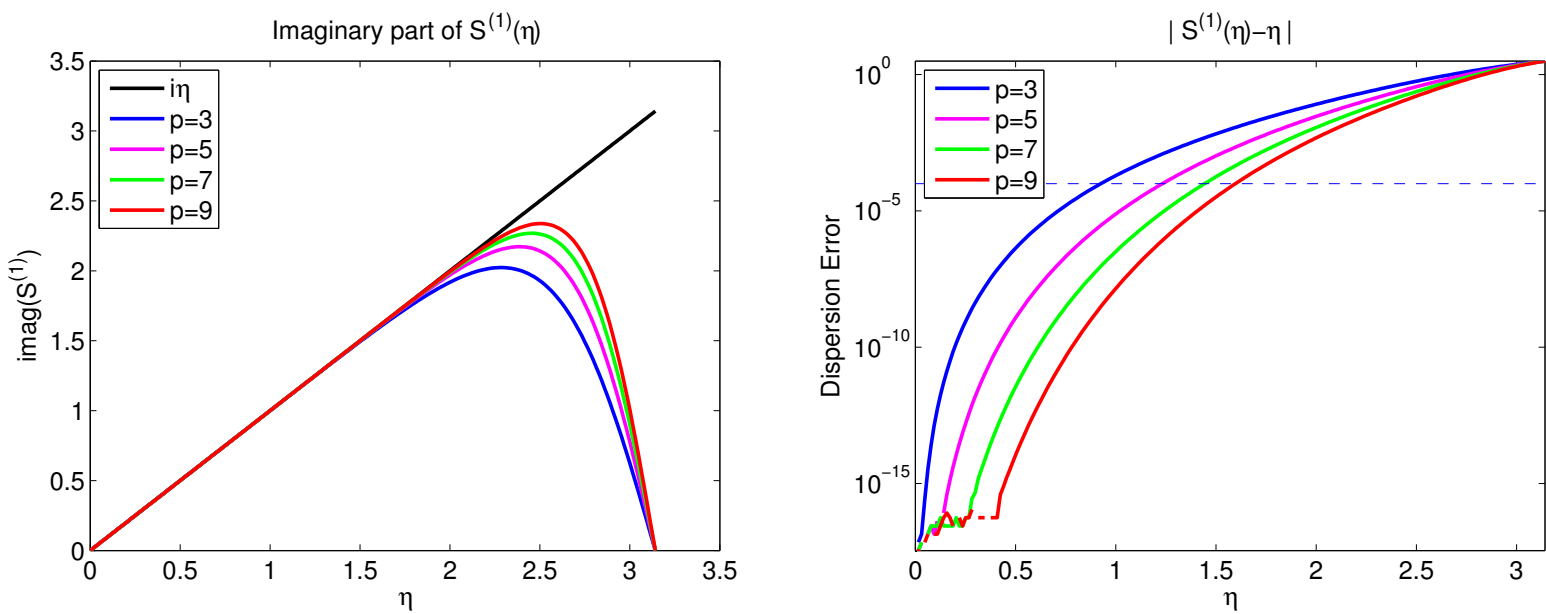

Figure 7: Symbols and dispersion errors of the first order difference operators for various $p$. Note we show the curves only for positive wave number as the negative wave numbers are determined via odd symmetry.

\subsection{Spectrum of the difference operators}

It is obvious from Figure 7 that the eigenvalues of the symbol are bounded above by $\pi$. Thus we only study the effect of the generic boundary closures by computing the maximum eigenvalues for the full discretization on a domain with 200 points. Precisely we consider the approximation to the operator $\frac{d}{d x}$ with zero boundary condition at inflow. We have also computed the eigenvalues for the approximation to the system (46)-477) considered below with the same results for the spectral radius. In this case we find that the simple choice of the ghost basis yields satisfactory results even for $p$ up to 17, though there may still be advantages to using the extrapolation method; see Table 7 below.

It is worth noting that the continuous operator with this boundary condition has no spectrum, but we expect the discrete eigenvalues to have both real and imaginary parts. We plot them for $p=3,7$ in Figure 8 


\begin{tabular}{|c|c|c|c|c|c|c|c|c|}
\hline$p$ & 3 & 5 & 7 & 9 & 11 & 13 & 15 & 17 \\
\hline \hline$h \rho\left(\left(M^{(p, G)}\right)^{-1} K^{(1, p, G)}\right)$ & 2.02 & 2.17 & 3.41 & 3.18 & 3.67 & 3.81 & 3.57 & 3.84 \\
\hline$E^{G}$ & 1.56 & 1.45 & 0.92 & 0.99 & 0.86 & 0.82 & 0.88 & 0.82 \\
\hline$h \rho\left(\left(M^{(p, X)}\right)^{-1} K^{(1, p, X)}\right)$ & 2.02 & 2.17 & 2.27 & 2.33 & 2.39 & 2.43 & 2.46 & 2.49 \\
\hline$E^{X}$ & 1.56 & 1.45 & 1.38 & 1.35 & 1.31 & 1.29 & 1.28 & 1.26 \\
\hline
\end{tabular}

Table 7: Table of maximum eigenvalues and normalized stability constraint for first derivatives with the ghost, G, and extrapolation, $\mathrm{X}$, basis closures at various orders.

By way of comparison we also plot the eigenvalues of the approximation to spatial operator in (46)- 47.) In that case the continuous problem has purely imaginary eigenvalues, but our upwind treatment of the boundary conditions leads to discrete eigenvalues with both real and imaginary parts. It is remarkable that the spectral radii are the same in the two cases, while a glance at the graphs shows that the cause is always a spurious eigenvalue arising in both approximations.
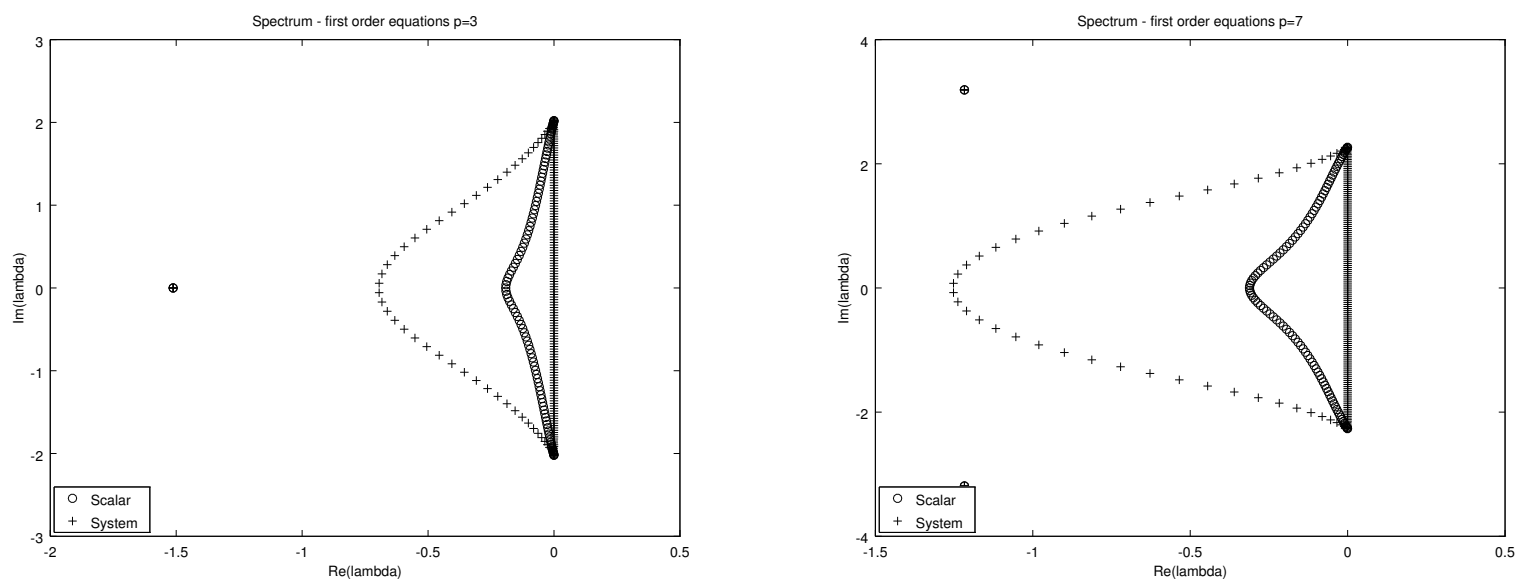

Figure 8: Spectra of the discrete spatial operators for approximations to $d / d x$ and the system 46-47) $p=3$ and $p=7$ with the ghost basis closure.

\subsection{A numerical experiment}

Lastly, we carry out a numerical experiment to evaluate the convergence of the full Galerkin difference discretizations for the first-order system. Here we consider the system

$$
\begin{aligned}
u_{t}+u_{x} & =0, & & v_{t}-v_{x}=0 \\
u(0, t)+v(0, t) & =0, & & u(1, t)-v(1, t)=0,
\end{aligned}
$$

with initial data leading to the exact solution

$$
\begin{aligned}
& u(x, t)=\sin \left(\frac{41}{2} \pi x\right) \cdot \cos \left(\frac{41}{2} \pi t\right)-\cos \left(\frac{41}{2} \pi x\right) \cdot \sin \left(\frac{41}{2} \pi t\right) \\
& v(x, t)=\sin \left(\frac{41}{2} \pi x\right) \cdot \cos \left(\frac{41}{2} \pi t\right)+\cos \left(\frac{41}{2} \pi x\right) \cdot \sin \left(\frac{41}{2} \pi t\right) .
\end{aligned}
$$

Similar to the experiments reported above for second order problems, we time step using a Runge-KuttaTaylor method of order $2 p+2$ but with $\Delta t / h=2 / 3$. As before, we choose such a high-order time integrator to be certain to observe any superconvergence associated with the accurate dispersion relation. 
Figure 9 shows the convergence history in the $L^{2}$-norm at the final time $t_{f}=100.5$ for both the ghost basis and extrapolation closures for $p=3,5,7,9$. The number of points in the domain for the refinement studies are $n=80: 20: 360$ for $p=3, n=60: 20: 340$ for $p=5, n=50: 15: 260$ for $p=7$, and $n=40: 15: 250$ for $p=9$. One plot is provided for each order, and reference lines are included which indicate the superconvergent rate $O\left(h^{2 p+2}\right)$ and the rate $O\left(h^{p+1}\right)$. Clearly at the early stages we observe superconvergence which relaxes to convergence closer to the prediction of Theorem 2 as the error becomes very small. We also note that, as for the second-order system, we see that the errors were somewhat lower for the ghost basis, particularly for fine meshes. This behavior may be related to the fact that that we initialize the solution at the ghost nodes very accurately using an extension of the exact solution evaluated at the ghost node locations.
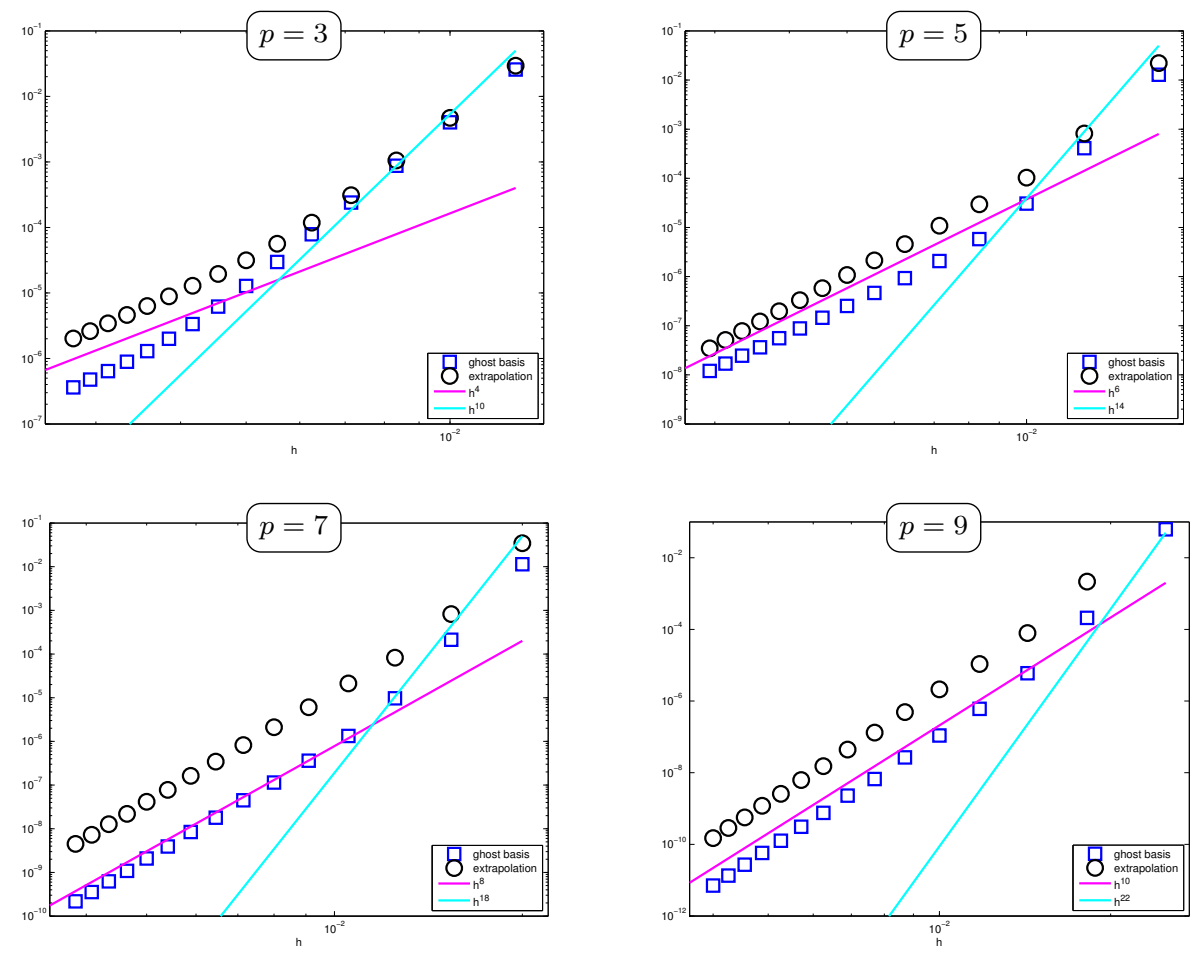

Figure 9: Results for convergence studies for the first-order system using the $L^{2}$-norm for $p=3,5,7,9$. The plots reflect the numerical errors in both the ghost basis and extrapolation methods, as well as reference lines indicating convergence rates of $O\left(h^{2 p+2}\right) O\left(h^{p+1}\right)$.

\section{Multiple dimensions and mapped grids}

The extension of the methods described above to Cartesian grids in multiple dimensions is straightforward. For simplicity suppose that we are solving the scalar wave equation with Neumann boundary conditions in $2+1$ dimensions

$$
u_{t t}=c^{2} u_{x x}+c^{2} u_{y y}+f(x, y, t),
$$

for $(x, y) \in\left(x_{a}, x_{b}\right) \times\left(y_{a}, y_{b}\right)$ with boundary conditions

$$
\begin{aligned}
& u_{x}\left(x=x_{a}, y, t\right)=0, \\
& u_{x}\left(x=x_{b}, y, t\right)=0, \\
& u_{y}\left(x, y=y_{a}, t\right)=0, \\
& u_{y}\left(x, y=y_{b}, t\right)=0 .
\end{aligned}
$$


Note that similar considerations would apply to the solution of systems in first order form. Introducing uniform grids, $x_{j}=x_{a}+j h_{x}, y_{k}=y_{a}+k h_{y}$, we may then simply construct Galerkin difference methods from the basis function ${ }^{3}$

$$
\psi_{j k}^{(p)}(x, y)=\phi_{j}^{(p)}(x) \cdot \phi_{k}^{(p)}(y) .
$$

We then find that the mass and stiffness matrices are simply expressed as tensor products of the mass and stiffness matrices constructed above. For example

$$
\begin{aligned}
M_{(j, k),(l, m)}^{(p)} & =\int_{x_{a}}^{x_{b}} \int_{y_{a}}^{y_{b}} \psi_{j k}^{(p)}(x, y) \psi_{l m}^{(p)}(x, y) d x d y \\
& =\int_{x_{a}}^{x_{b}} \phi_{j}^{(p)}(x) \phi_{l}^{(p)}(x) d x \cdot \int_{y_{a}}^{y_{b}} \phi_{k}^{(p)}(y) \phi_{m}^{(p)}(y) d y \\
& =M_{j l}^{(x, p)} M_{k m}^{(y, p)},
\end{aligned}
$$

or using tensor product notation

$$
M^{(2, p)}=M^{(x, p)} \otimes M^{(y, p)} .
$$

Repeating for the stiffness matrices we derive the semidiscrete system:

$$
M^{(x, p)} \otimes M^{(y, p)} \frac{d^{2} U}{d t^{2}}=\left(K^{(x, 2, p)} \otimes M^{(y, p)}+M^{(x, p)} \otimes K^{(y, 2, p)}\right) U+F .
$$

The essential point is that by leveraging the tensor product structure of the mass matrix, the direct solution to the system (54) can be found through a series of solutions to banded linear systems corresponding to lines of constant $x$ and $y$. Therefore, the direct solution of the system will still scale linearly in the number of grid points.

Of course in general one must solve problems in mapped domains to account for any nontrivial geometry. In this case we must solve the transformed system for $\tilde{u}(r, s, t)=u(X(r, s), Y(r, s), t)$ which takes the form [24, Ch. 2]

$$
J \frac{\partial^{2} \tilde{u}}{\partial t^{2}}=\frac{\partial}{\partial r}\left(\alpha \frac{\partial \tilde{u}}{\partial r}\right)+\frac{\partial}{\partial s}\left(\beta \frac{\partial \tilde{u}}{\partial r}\right)+\frac{\partial}{\partial r}\left(\beta \frac{\partial \tilde{u}}{\partial s}\right)+\frac{\partial}{\partial s}\left(\gamma \frac{\partial \tilde{u}}{\partial s}\right)+J F
$$

where $J(r, s)$ is the Jacobian matrix and $\alpha(r, s), \beta(r, s)$, and $\gamma(r, s)$ are smooth functions determined by the coordinate transformations. The direct construction of the Galerkin difference method would lead to mass and stiffness matrices which would still be banded, but which would no longer maintain their tensor product structure. As a result, the direct solution of the linear system determining the time derivatives would no longer be linear in the number of grid points, and thus would dominate the cost. We propose to circumvent this difficulty using an idea proposed by Warburton [25]; namely to replace the basis functions $\psi_{j k}^{(p)}(r, s)$ by

$$
\tilde{\psi}_{j k}^{(p)}(r, s)=J^{-1 / 2}(r, s) \phi_{j}^{(p)}(r) \cdot \phi_{k}^{(p)}(s) .
$$

Then the tensor-product structure of the mass matrix is retained. (A tensor-product structure for the stiffness matrices is not essential as they are not factored.)

Lastly we note that we have not studied the superconvergence of the dispersion error for problems with variable coefficients, as required by the domain mappings. However, in analogy with the results for B-splines proven in [15, we expect they will still hold. Experiments with the proposed method will be reported elsewhere.

\section{Conclusion}

In this manuscript we have explored the basic properties of Galerkin difference methods along with various boundary closure strategies. The schemes are built in the framework of a traditional finite element method and employ a weak form of the operator and a Galerkin projection onto a basis. Unlike traditional

\footnotetext{
${ }^{3}$ More generally different polynomial degrees could be used in different coordinate directions.
} 
approaches, these methods do not introduce interior degrees of freedom and obtain high order accuracy by including neighboring DOFs while imposing only low-order continuity at element boundaries. In this sense the basis functions are derived using principles familiar to the finite difference community. Two generic boundary closures, ghost basis and extrapolation, were discussed, as well as a boundary closure based on compatibility. Numerical experiments illustrated the properties of these approaches for hyperbolic PDEs in both first and second-order form. In our view this overall methodology provides a natural approach to the derivation of energy-stable difference methods. In particular GD discretizations can be automatically constructed to achieve any desired order of accuracy, employ full-order $O\left(h^{p+1}\right)$ boundary closures, and exhibit only moderate growth in the spectral radius of the discrete operator. These tasks are more difficult to accomplish using the usual SBP technology, though we do note that progress has been made in stabilizing methods with boundary closures leading to the full accuracy of the interior differencing and with coupling on hybrid grids [26. A potential defect of the GD method is an increase in bandwidth relative to standard difference methods using the same polynomial approximations at the nodes. However this is compensated for by the fact that the dispersion error in GD methods is accurate to very high order.

There are a number of possibilities for extension of these ideas to complex geometry. For example, directly coupling the schemes to standard finite element methods on hybrid grids appears to be straight forward. Alternately one might explore multiblock grids, overlapping grids, or embedded boundaries. Finally, note that the approach followed in Section 2 in deriving basis functions is generally applicable to any global reconstruction from discrete data. Therefore, in principle the GD approach could be generalized directly to arbitrary grids. Each of these is a potential avenue for future research. In addition we intend to examine the effects of mass-lumping, based for example on Alpert's hybrid Gauss-trapezoid rules [27, to diagonalize the mass-matrix and reduce the bandwidth of the stiffness matrices away from boundaries. Finally, as the GD approach matures a thorough comparison with existing schemes, including summation-by-parts operators and traditional finite element methods, should be pursued. 


\section{References}

[1] B. Sjögreen, N. A. Petersson, A fourth order finite difference scheme for the elastic wave equation in second order formulation, Scient. Comput. 52 (2012) 17-48.

[2] B. Strand, Summation by parts for finite difference approximations for d/dx, J. Comput. Phys. 110 (1994) 47-67.

[3] M. Svärd, J. Nordström, Review of summation-by-parts schemes for initial-boundary-value problems, J. Comput. Phys. 268 (2014) 17-38.

[4] T. R. Hughes, The Finite Element Method: Linear Static and Dynamic Finite Element Analysis, Dover Publications, New York, 2000.

[5] G. Strang, G. Fix, An Analysis of the Finite Element Method 2nd Edition, 2nd Edition, WellesleyCambridge, 2008.

[6] P. Solin, K. Segeth, I. Dolezel, Higher-Order Finite Element Methods, CRC-Press, 2004.

[7] J. S. Hesthaven, T. Warburton, Nodal Discontinuous Galerkin Methods: Algorithms, Analysis, and Applications., Springer Verlag, New York, 2008.

[8] A. Patera, A spectral element method for fluid dynamics: Laminar flow in a channel expansion, J. Comput. Phys. 54 (1984) 468-488.

[9] T. Hughes, J. A. Cottrell, Y. Bazilevs, Isogeometric analysis: CAD, finite elements, NURBS, exact geometry and mesh refinement, Comput. Method. Appl. Mech. Engrg. 194 (2005) 4135-4195.

[10] K. Kormann, M. Kronbichler, B. Müller, Derivation of strictly high order difference approximations for variable-coefficient PDE, J. Sci. Comput. 50 (2012) 167-197.

[11] S. Lele, Compact finite difference schemes with spectral-like resolution, J. Comput. Phys. 103 (1992) $16-42$.

[12] T. Warburton, T. Hagstrom, Taming the CFL number for discontinuous Galerkin methods on structured meshes, SIAM J. Numer. Anal. 46 (6) (2008) 3151-3180.

[13] S. Ghader, J. Nordström, High-order compact finite difference schemes for the vorticity-divergence representation of the spherical shallow water equations, Int. J. Numer. Meth. Fl. 78 (2015) 709-738.

[14] B. Swartz, B. Wendroff, The relative efficiency of finite difference and finite element methods. I: hyperbolic problems and splines, SIAM J. Numer. Anal. 11 (1974) 979-993.

[15] V. Thomée, B. Wendroff, Convergence estimates for Galerkin methods for variable coefficient initial value problems, SIAM J. Numer. Anal. 11 (1974) 1059-1068.

[16] T. Hagstrom, J. Banks, Galerkin difference methods from band-limited interpolation functions, in: 12th International Conference on Mathematical and Numerical Aspects of Wave Propagation, Karlsruhe, 2015.

[17] W. D. Henshaw, A high-order accurate parallel solver for Maxwell's equations on overlapping grids, SIAM J. Sci. Comput. 28 (5) (2006) 1730-1765.

[18] G. Baker, Error estimates for finite element methods for second order hyperbolic equations, SIAM J. Num. Anal. 13 (1976) 564-576.

[19] J.-L. Guermond, R. Pasquetti, A correction technique for the dispersive effects of mass lumping for transport problems, Computer Meth. Appl. Mech. Engrg. 253 (2012) 186-198. 
[20] D. Gottlieb, B. Gustafsson, P. Olsson, B. Strand, On the superconvergence of Galerkin methods for hyperbolic IBVP, SIAM J. Numer. Anal. 33 (1996) 1778-1796.

[21] J. Hesthaven, T. Warburton, Nodal Discontinuous Galerkin Methods, no. 54 in Texts in Applied Mathematics, Springer-Verlag, New York, 2008.

[22] X. Meng, C.-W. Shu, B. Wu, Optimal error estimates for discontinuous Galerkin methods based on upwind-biased fluxes for linear hyperbolic equations, Math. Comp.To appear.

[23] P. Ciarlet, The finite element method for elliptic problems, Classics in Applied Mathematics 40, SIAM, Philadelphia, 2002.

[24] P. Knupp, S. Steinberg, Fundamentals of Grid Generation, CRC Press, Boca Raton, 1993.

[25] T. Warburton, A low-storage curvilinear discontinuous Galerkin method for wave problems, SIAM J. Sci. Comput. 35 (2013) A1987-A2012.

[26] J. Kozdon, L. Wilcox, Provably stable, general purpose projection operators for high-order finite difference methods, preprint (2015).

[27] B. Alpert, Hybrid Gauss-trapezoidal rules, SIAM J. Sci. Comp. 20 (1999) 1551-1584. 